\title{
Recent Results from PHOBOS at RHIC
}

\author{
B.B.Back ${ }^{1}$, M.D.Baker ${ }^{2}$, M.Ballintijn ${ }^{4}$, D.S.Barton ${ }^{2}$, B.Becker ${ }^{2}$, R.R.Betts ${ }^{6}$, A.A.Bickley ${ }^{7}$, \\ R.Bindel $^{7}$, A.Budzanowski ${ }^{3}$, W.Busza ${ }^{4}$, A.Carroll ${ }^{2}$, M.P.Decowski ${ }^{4}$, E.García $^{6}$, \\ T.Gburek $^{3}$, N.George ${ }^{1,2}$, K.Gulbrandsen ${ }^{4}$, S.Gushue ${ }^{2}$, C.Halliwell ${ }^{6}$, J.Hamblen ${ }^{8}$, \\ A.S.Harrington ${ }^{8}$, G.A.Heintzelman ${ }^{2}$, C.Henderson ${ }^{4}$, D.J.Hofman ${ }^{6}$, R.S.Hollis ${ }^{6}$, R.Hoyński ${ }^{3}$, \\ B.Holzman ${ }^{2}$, A.Iordanova ${ }^{6}$, E.Johnson ${ }^{8}$, J.L.Kane ${ }^{4}$, J.Katzy ${ }^{4,6}$, N.Khan ${ }^{8}$,

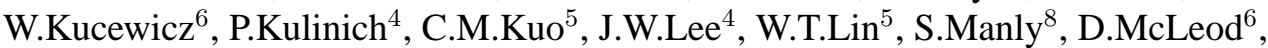 \\ A.C.Mignerey ${ }^{7}$, R.Nouicer ${ }^{2,6}$, A.Olszewski ${ }^{3}$, R.Pak ${ }^{2}$, I.C.Park ${ }^{8}$, H.Pernegger ${ }^{4}$, \\ C.Reed ${ }^{4}$, L.P.Remsberg ${ }^{2}$, M.Reuter ${ }^{6}$, C.Roland ${ }^{4}$, G.Roland ${ }^{4}$, L.Rosenberg ${ }^{4}$,

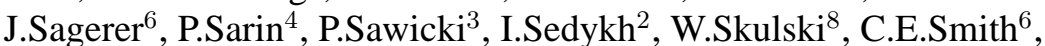 \\ P.Steinberg ${ }^{2}$, G.S.F.Stephans ${ }^{4}$, A.Sukhanov ${ }^{2}$, J.-L.Tang ${ }^{5}$, M.B.Tonjes ${ }^{7}$, A.Trzupek ${ }^{3}$, \\ C.Vale ${ }^{4}$, G.J.van Nieuwenhuizen ${ }^{4}$, R.Verdier ${ }^{4}$, G.I.Veres ${ }^{4}$, F.L.H.Wolfs ${ }^{8}$, B.Wosiek ${ }^{3}$, \\ K.Woźniak ${ }^{3}$, A.H.Wuosmaa ${ }^{1}$, B.Wysouch ${ }^{4}$, and J.Zhang ${ }^{4}$ \\ ${ }^{1}$ Physics Division, Argonne National Laboratory, Argonne, IL 60439-4843, USA \\ ${ }^{2}$ Chemistry and C-A Departments, Brookhaven National Laboratory, Upton, NY 11973-5000, USA \\ ${ }^{3}$ Institute of Nuclear Physics, Kraków, Poland \\ ${ }^{4}$ Laboratory for Nuclear Science, Massachusetts Institute of Technology, Cambridge, MA 02139-4307, USA \\ ${ }^{5}$ Department of Physics, National Central University, Chung-Li, Taiwan \\ ${ }^{6}$ Department of Physics, University of Illinois at Chicago, Chicago, IL 60607-7059, USA \\ ${ }^{7}$ Department of Chemistry, University of Maryland,College Park, MD 20742, USA \\ ${ }^{8}$ Department of Physics and Astronomy, University of Rochester, Rochester,NY 14627, USA
}

Received on 26 September, 2003

\begin{abstract}
PHOBOS is one of the four heavy ion experiments currently running at the Relativistic Heavy Ion Collider (RHIC). In this paper we will present some of the current results from PHOBOS: the study of charged multiplicity distribution as function of pseudorapidity and centrality at $\sqrt{s_{N N}}=19.6,130$ and $200 \mathrm{GeV}$, a discussion of multiplicity scaling, and a measurement of transverse momentum spectra $\left(p_{T}\right)$ of charged hadrons produced in $\mathrm{d}+\mathrm{Au}$ and $\mathrm{Au}+\mathrm{Au}$ interactions at $\sqrt{s_{N N}}=200 \mathrm{GeV}$. The dependence of the Au+Au yields as function of centrality is found to be independent of $p_{T}$. A strong suppression in the $\mathrm{Au}+\mathrm{Au}$ hadron spectra relative to $\mathrm{p} \overline{\mathrm{p}}$ at high $p_{T}$ is observed. The spectral shape for $\mathrm{d}+\mathrm{Au}$ collisions is significantly different from the observations in $\mathrm{Au}+\mathrm{Au}$, the yield suppression is not observed in $\mathrm{d}+\mathrm{Au}$.
\end{abstract}

The fundamental theory of strong interactions predicts that a Quark-Gluon Plasma (QGP) will be formed in hadronic systems at sufficiently high energy density [1]. The QGP is the state of matter that is believed to have existed about 10 micro-seconds after the Big Bang, and is a conglomerate of free quarks and gluons. The goal of the relativistic heavy ion physics program over the past two decades has been to find signatures of the formation of the QGP. From data collected at the AGS, the SPS and so far from RHIC, it is clear that the search for the QGP requires a systematic effort of the entire heavy ion physics community. In this paper we present PHOBOS' contribution to this task. The data described in this paper were taken during the RHIC 2000 run, $\mathrm{Au}+\mathrm{Au}$ at $\sqrt{s_{N N}}=19.6$ and $130 \mathrm{GeV}$, the 2001 $\mathrm{Au}+\mathrm{Au}$ run at $\sqrt{s_{N N}}=200 \mathrm{GeV}$, and the $2003 \mathrm{~d}+\mathrm{Au}$ run at $\sqrt{s_{N N}}=200 \mathrm{GeV}$. A full description of the PHOBOS detector used during these runs as well a description of the event selection process can be found at $[2,3]$.

One of the important contributions of PHOBOS to the field is the measurement of charged particle multiplicity as over a large pseudorapidity $(\eta)$ coverage. Fig. 1 summarizes all the $\mathrm{Au}+\mathrm{Au}$ multiplicity measurements taken by PHOBOS. Details of the analysis procedures can be found in Ref. [4]. The centrality of the collisions is characterized by the average number of participants $\left(N_{\text {part }}\right)$. For the 130 and $200 \mathrm{GeV}$ data sets this was estimated from the data using two sets of 16 plastic scintillator counters (the "paddle" counters), with acceptance of $3<|\eta|<4$.5. It was found that the truncated mean of the ADC signals from these counters is proportional to the charged multiplicity in their acceptance. $N_{\text {part }}$ is then extracted for a specified fraction of the cross-section from the truncated mean, assuming that this signal increases monotonically with centrality. The 


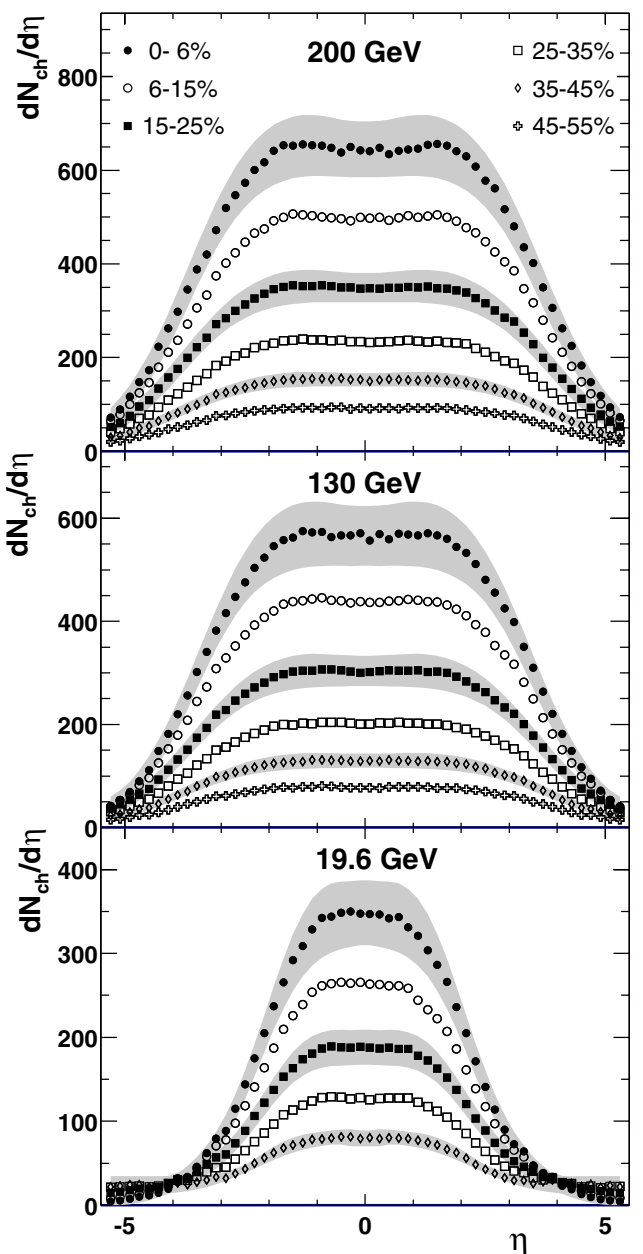

Figure 1. Charged particle pseudorapidity distributions, $d N_{c h} / d \eta$, measured for $\mathrm{Au}+\mathrm{Au}$ at $\sqrt{s_{N N}}=200,130$, and $19.6 \mathrm{GeV}$ for the specified centrality bins. These bins range from $0-6 \%$ central to $45-55 \%$ in the case of the higher energy data and $0-6 \%$ to 35 $45 \%$ for the $19.6 \mathrm{GeV}$ data. Statistical errors are negligible and typical systematic errors (90\% C.L.) are shown as shaded bands for selected centrality bins.

monotonic relation was verified using the correlation between the paddle counters and the zero degree calorimeters. The calculation of $N_{\text {part }}$ and the associated systematic errors is outlined in Ref [5]. For the $\sqrt{s_{N N}}=19.6 \mathrm{GeV}$ data set, the lower beam rapidity precludes use of this method; instead a different quantity was constructed "EOCT". This quantity is proportional to the path-length corrected sum of the energy deposited in the octagon detector. As for the paddle counters, it is assumed only that EOCT varies monotonically with $N_{\text {part }}$. Finally, for the d+Au data set, the silicon ring detectors were use in a similar way to determine centrality bins.

An empirical observation derived from the multiplicity measurements is the scaling of the multiplicity distributions with $N_{\text {part }}$. Fig. 2 , shows the total charged multiplicity per participant pair as a function of the number of participants for $\mathrm{Au}+\mathrm{Au}$ at three different energies. Also shown in the figure are results for $\mathrm{p} \overline{\mathrm{p}}$ and $e^{+} e^{-}$systems at the same value

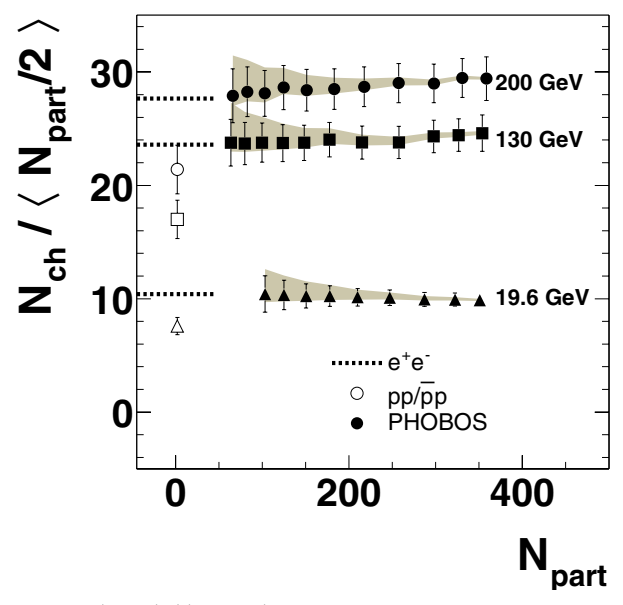

Figure 2. $\left\langle N_{c h}\right\rangle /\left\langle N_{\text {part }}\right\rangle$ vs. $N_{\text {part }}$ for $\sqrt{s_{N N}}=19.6,130$, and $200 \mathrm{GeV}$ (closed symbols). The error includes contributions from the uncertainty on overall $N_{c h}$ scale and $N_{\text {part }}$ scale. The shaded band shows the uncertainty on the extrapolation procedure for larger $\eta$. The open symbols show UA1 p p data at $200 \mathrm{GeV}$ and interpolated results for the lower energies. The dotted lines show the values for $e^{+} e^{-}$.

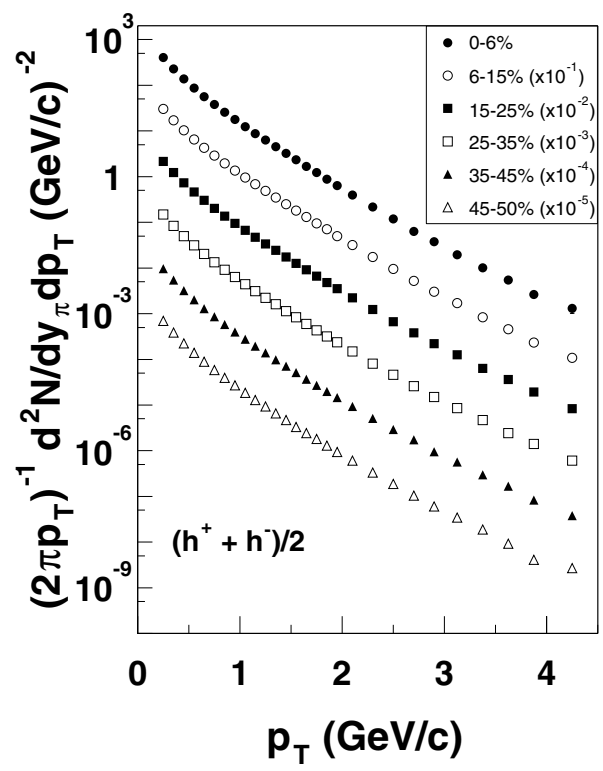

Figure 3. Invariant yields of charged hadrons for $\sqrt{s_{N N}}=200$ $\mathrm{GeV} \mathrm{Au}+\mathrm{Au}$ collisions as a function of $p_{T}$ for 6 centrality bins. For clarity, consecutive bins are scaled by factors of 10 .

of $\sqrt{s}$. The total multiplicity in $\mathrm{Au}+\mathrm{Au}$ scales approximately with the number of participants and is the same as the production for $e^{+} e^{-}$at the same energy. On the other hand, production in $\mathrm{p} \overline{\mathrm{p}}$ is reduced, in comparison to $\mathrm{Au}+$ $\mathrm{Au}$. A detailed discussion on this scaling behavior is found in Ref. [6].

In Fig. 3, we present the invariant cross-section of charged hadrons, obtained by averaging the yields of positive 


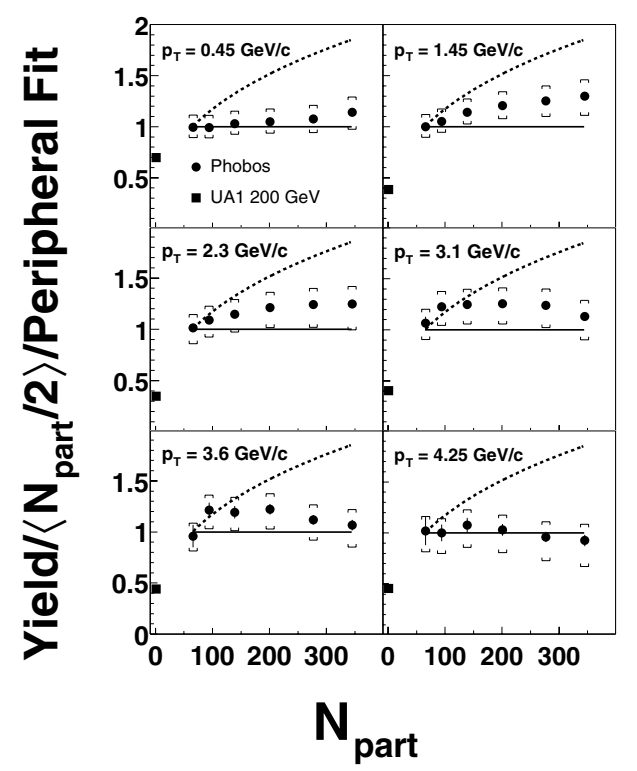

Figure 4. Charged hadron yields per participant pair in 6 different transverse momentum bins, plotted as a function of $N_{\text {part }}$. The data are normalized to the yield in the most peripheral centrality bin. The dashed (solid) line shows the expectation for $N_{\text {coll }}$ $\left(N_{\text {part }}\right)$ scaling from peripheral to central collisions. The brackets indicate the systematic uncertainty for the centrality evolution of this ratio at the $90 \%$ C.L.

and negative hadrons as a function of transverse momentum for $\sqrt{s_{N N}}=200 \mathrm{GeV} \mathrm{Au+Au}$ collisions. Data are shown for 6 centrality bins, ranging from $\left\langle N_{\text {part }}\right\rangle=58$ to $\left\langle N_{\text {part }}\right\rangle=344$. The spectra exhibit a smooth fall with increasing $p_{T}$, with a slightly concave shape. Details of the analysis and a broader discussion of the $p_{T}$ spectra are in Ref. [2].

In order to study the evolution of the spectra with centrality in detail Fig. 4 shows the multiplicity yield per participant pair for six $p_{T}$ values, divided by a fit to the most peripheral bin plotted as a function of the number of participants. For reference $p \bar{p}$ data are included in the plot. The dashed and solid lines represent the expectation for $\left\langle N_{\text {coll }}\right\rangle$ and $\left\langle N_{\text {part }}\right\rangle$ scaling. The data seem to scale closer to $\left\langle N_{\text {part }}\right\rangle$ than to $\left\langle N_{\text {coll }}\right\rangle$ for all $p_{T}$ bins and centralities. We also observe surprisingly that the spectra do not depend on centrality.

Figure 5 shows the yield per participant pair divided by a fit to the invariant cross section for proton-antiproton collisions (200 GeV UA1) plotted as a function of $p_{T}$, for the most peripheral and most central bin. The dashed and solid lines show the expectation of $\left\langle N_{\text {coll }}\right\rangle$ and $\left\langle N_{\text {part }}\right\rangle$ scaling. The brackets show the systematic uncertainty. We can see, again, that the general shape of the curves is only weakly dependent on the centrality and that, for $p_{T}$ values up to 2 $\mathrm{GeV} / \mathrm{c}$ there is an increase of the yield in $\mathrm{Au}+\mathrm{Au}$ collisions compared to $\mathrm{p} \overline{\mathrm{p}}$. After $2 \mathrm{GeV} / \mathrm{c}$ the relative yield decreases, for all centrality bins.

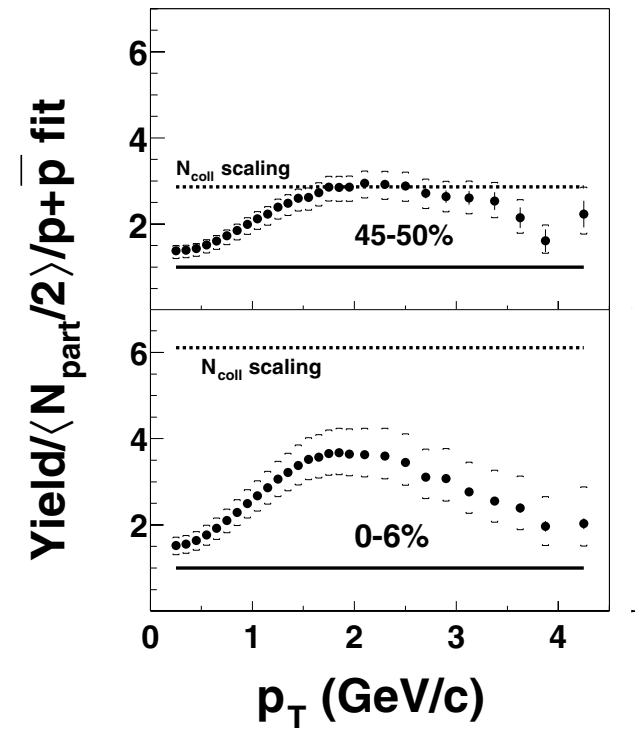

Figure 5. Ratio of the yield of charged hadrons in $\mathrm{Au}+\mathrm{Au}$ as a function of $p_{T}$ for the most peripheral and the most central bin to a fit of proton antiproton data scaled by $\left\langle N_{\text {part }} / 2\right\rangle$. The dashed(solid) line shows the expectation of $N_{\text {coll }}\left(N_{\text {part }}\right)$ scaling relative to $\mathrm{p} \overline{\mathrm{p}}$ collisions. The brackets show the systematic uncertainty.

Two possible explanations have been suggested for the this yield "quenching". The first ("initial state") model suggest that the effect is due to a modification of the wave functions of the nucleons in the colliding ions. This produces an effective increase of the interaction length in such way that the nucleons in an ion will interact coherently with all the nucleons in the other ion in the longitudinal dimension [7]. This model predicts not only the yield quenching at high $p_{T}$, but also the scaling of the yield with the number of participants. The second ("final state") model suggests that the suppression comes from an increase of the energy loss of the partons traveling through the hot dense medium formed in the $\mathrm{Au}+\mathrm{Au}$ collisions [8]. One way to discriminate between these models is to reduce the size of the hot and dense medium, by colliding $\mathrm{d}+\mathrm{Au}$ instead of $\mathrm{Au}+\mathrm{Au}$. RHIC's 2003 run was dedicated mainly to d+Au collisions at $\sqrt{s_{N N}}=200 \mathrm{Gev}$. Fig. 6 shows the measured invariant yield of charged hadrons for $\mathrm{d}+\mathrm{Au}$ collisions as function of $p_{T}$. Details of the analysis and a broader discussion of $p_{T}$ spectra are given in Ref. [3].

In Fig. 7 we present the nuclear modification factor $R_{d A u}$ as function of $p_{T}$ for four centrality bins defined as the ratio of the $\mathrm{d}+\mathrm{Au}$ invariant cross section divided by the $\mathrm{p} \overline{\mathrm{p}}$ UA1 yield, scaled by the proton-antiproton invariant cross section $(41 \mathrm{mb})$ and $\left\langle N_{\text {coll }}\right\rangle$. For all the centrality bins we observe a rapid rise of $R_{d A u}$ from low $p_{T}$ reaching a maximum at $2 \mathrm{GeV} / \mathrm{c}$. For comparison this factor is also plotted for the most central bin from central $\mathrm{Au}+\mathrm{Au}$ collisions at $\sqrt{s_{N N}}=200 \mathrm{GeV}$. In striking contrast to the behavior of $R_{d A u}, R_{A u A u}$ also increases initialy as function of $p_{T}$ but after $2 \mathrm{GeV} / \mathrm{c}$ decreases sharply at larger $p_{T}$ values. 


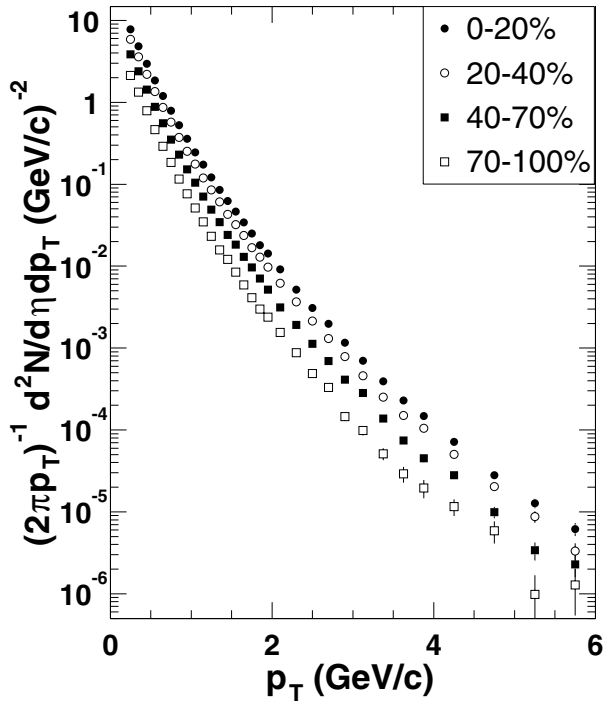

Figure 6. Invariant yields of charged hadrons for $\sqrt{s_{N N}}=200$ $\mathrm{GeV} \mathrm{d}+\mathrm{Au}$ collisions as a function of $p_{T}$ for 4 centrality bins. Only statistical errors are shown.

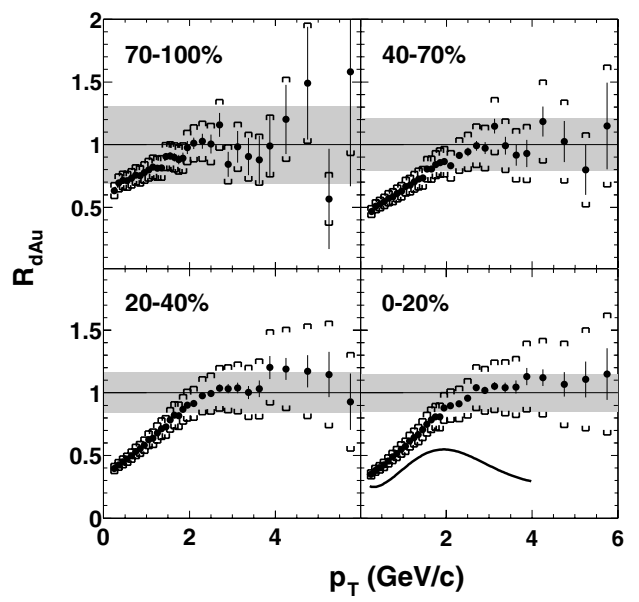

Figure 7. Nuclear modification factor $R_{d A u}$ as a function of $p_{T}$ for four centrality bins. For the most central bin, the spectral shape for central $\mathrm{Au}+\mathrm{Au}$ data relative to $p+\bar{p}$ is shown for comparison. The shaded area shows the uncertainty in $R_{d A u}$ due to the systematic uncertainty in $\left\langle N_{\text {coll }}\right\rangle$ and the UA1 scale error ( $90 \%$ C.L.). The brackets show the systematic uncertainty of the $\mathrm{d}+\mathrm{Au}$ spectra measurement (90\% C.L.).

In Fig. 8 the nuclear modification factor as a function of $\left\langle N_{\text {coll }}\right\rangle$ in four $p_{T}$ bins is presented. The "initial state" parton saturation model predicts a decrease of 25 $30 \%$ for $R_{d A u}$ for $\left\langle N_{\text {coll }}\right\rangle \geq 6$. At $p_{T}=4 \mathrm{GeV} / \mathrm{c}$, $R_{d A u}\left(N_{\text {coll }}=14.6\right) / R_{d A u}\left(N_{\text {coll }}=5.4\right)=1.08 \pm 0.06$.
The data therefore disfavor the initial state interpretation of high- $p_{T}$ hadron suppression. Thus, the yield quenching of high $p_{T}$ in $\mathrm{Au}+\mathrm{Au}$ collisions can not be accounted by initial state interactions that should be also present in $\mathrm{d}+\mathrm{Au}$. The suppression of the inclusive yield observed in central $\mathrm{Au}+\mathrm{Au}$ is consistent with final-state interactions with a dense medium generated in such collisions.

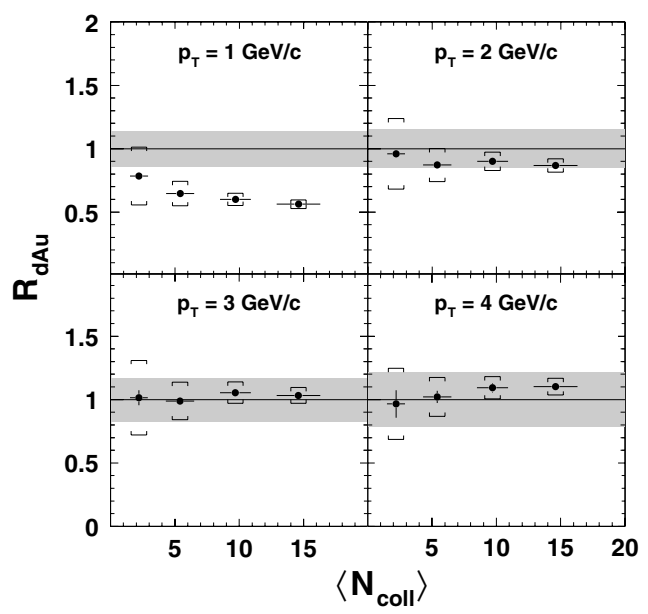

Figure 8. Nuclear modification factor $R_{d A u}$ as a function of centrality in four bins of transverse momentum. The brackets indicate the point-to-point systematic error, dominated by the uncertainty in the number of collisions for each centrality bin. The grey band shows the overall scale uncertainty at each $p_{T}$. Systematic errors are at $90 \%$ C.L.

This work was partially supported by U.S. DOE grants DE-AC02-98CH10886, DE-FG02-93ER40802, DE-FC02-94ER40818, DE-FG02-94ER40865, DE-FG0299ER41099, and W-31-109-ENG-38, US NSF grants 9603486, 9722606 and 0072204, Polish KBN grant 2-P03B10323, and NSC of Taiwan contract NSC 89-2112-M-008024.

\section{References}

[1] J. P. Blaizot, Nucl.Phys. A661, 3 (1999).

[2] B. B. Back et al., hep-ex/0210006.

[3] B. B. Back et al., hep-ex/0306025.

[4] B. B. Back et al., Phys. Rev. Lett. 87, 102303 (2001).

[5] B. B. Back et al., Phys. Rev. C 65, 061901R (2002).

[6] B. B. Back et al., hep-ex/0301017.

[7] D. Kharzeev, E. Levin, and L. McLerran, hep-ex/0210332.

[8] M. Gyulassy, I. Vitev, X. Wang, and B. Zhang, hepex/0302077. 